\title{
Frontières
}

\section{La marginalité n'est pas une maladie}

\section{Mariane Matte}

Volume 14, numéro 1, automne 2001

Où est la marge ?

URI : https://id.erudit.org/iderudit/1074166ar

DOI : https://doi.org/10.7202/1074166ar

Aller au sommaire du numéro

Éditeur(s)

Université du Québec à Montréal

ISSN

1180-3479 (imprimé)

1916-0976 (numérique)

Découvrir la revue

Citer ce document

Matte, M. (2001). La marginalité n'est pas une maladie. Frontières, 14(1), 68-70. https://doi.org/10.7202/1074166ar d'utilisation que vous pouvez consulter en ligne.

https://apropos.erudit.org/fr/usagers/politique-dutilisation/ 


\section{La marginalité n'est pas une maladie}

\author{
Mariane Matte, \\ coordonnatrice au journal \\ Pusher d'Infos.
}

Je m'appelle Mariane. Il fut un temps où l'on disait de moi que j'étais la meilleure pipe en ville. Il fut un temps où je n'avais plus de veines.

Dès ma naissance, j'ai fait partie de ceux que l'on pointe du doigt ; une rareté dans un petit village au fin fond du Lac-Saint-Jean. J'étais une enfant adoptée. Un jour où je pleurais sur cette différence, ma mère m'a dit: "Sois fière d'avoir été adoptée, car nous, on t'a choisie. » J'ai donc commencé ma vie, différente et fière de l'être. Bébé d'une famille de trois enfants, tous adoptés. Chapeau à mes parents qui nous ont donné tout ce qu'un enfant peut espérer. J'évoluais dans un monde adulte, étant précoce et dégourdie.

Très jeune, je me souviens d'avoir éprouvé une grande tristesse après avoir regardé $L e$ merveilleux monde de Walt Disney. D'avoir consciemment réalisé que ma vie était plate et que mon avenir était voué à la platitude. J'avais besoin de me dégourdir, de mettre de l'aventure, de l'extraordinaire dans ma vie. Mon désir d'être différente s'accentuait d'année en année, comme si je ne pouvais être aimée si je n'étais pas différente. J'avais toujours besoin de me faire voir, de me démarquer. Je me suis associée d'instinct, dès le primaire aux rebelles. Prendre plaisir à désobéir, fumer en cachette, voler. Je suis devenue très vite un problème partout où j'allais. Dans les yeux de ma mère chérie, je restais une bonne fille qui n'avait que quelques mauvaises habitudes qui se régleraient avec le temps.

À l'âge de 12 ans, ma demi-sœur, mariée à un Espagnol, m'invite pour passer six semaines en Espagne. Ce voyage m'a donné le goût de l'aventure, la vraie et m'a ouvert les yeux sur autre chose que mon nombril. Je crois que la piqûre du voyage, avant celle de la dope, m’a sauvé la vie. Merci à vous Diane et Jaime.

À 16 ans, ma mère biologique fait irruption dans ma vie pour m'annoncer que je suis née à la suite d'un viol. Cette affirmation n'a fait que m'endurcir, m'a déconnectée davantage. J'avais l'inconscient d'un enfant hypothéqué par l'inceste d'un grandpère alcoolique et l'adolescence meurtrie par une conception violente et malsaine.

Aujourd'hui, je ne possède qu'un niveau scolaire de secondaire III, et j'ai fréquenté cinq écoles différentes. J'étais toujours à la recherche du voyage, de ce besoin de découvrir de nouveaux visages, de cette adaptation à l'inconnu. C'est ce qui m'a poussée à la marge, car j'ai développé à outrance cette facette de l'existence. Ce besoin constant d'adrénaline, de me surpasser et de toujours réussir à me faire aimer et accepter. Comme je m'ennuyais constamment, quoi de mieux que la dope; cette chère altération de l'esprit.

Est-ce que j'ai consommé pour oublier la souffrance? Je ne sais pas, mais avec le temps, j'ai souffert de consommer.

Mon histoire, je la commence à Montréal, car c'est ici que je suis montée très haut et retombée très bas. Comme toutes les filles qui commencent dans le monde de la cocaïne, c'est le pied. Le sentiment d'être terriblement $I N$, le jet-set quoi. Je me suis improvisée massothérapeute professionnelle. Je travaillais à Ville Mont-Royal et je découvrais pour la première fois, de façon consciente, mes forces de manipulation.

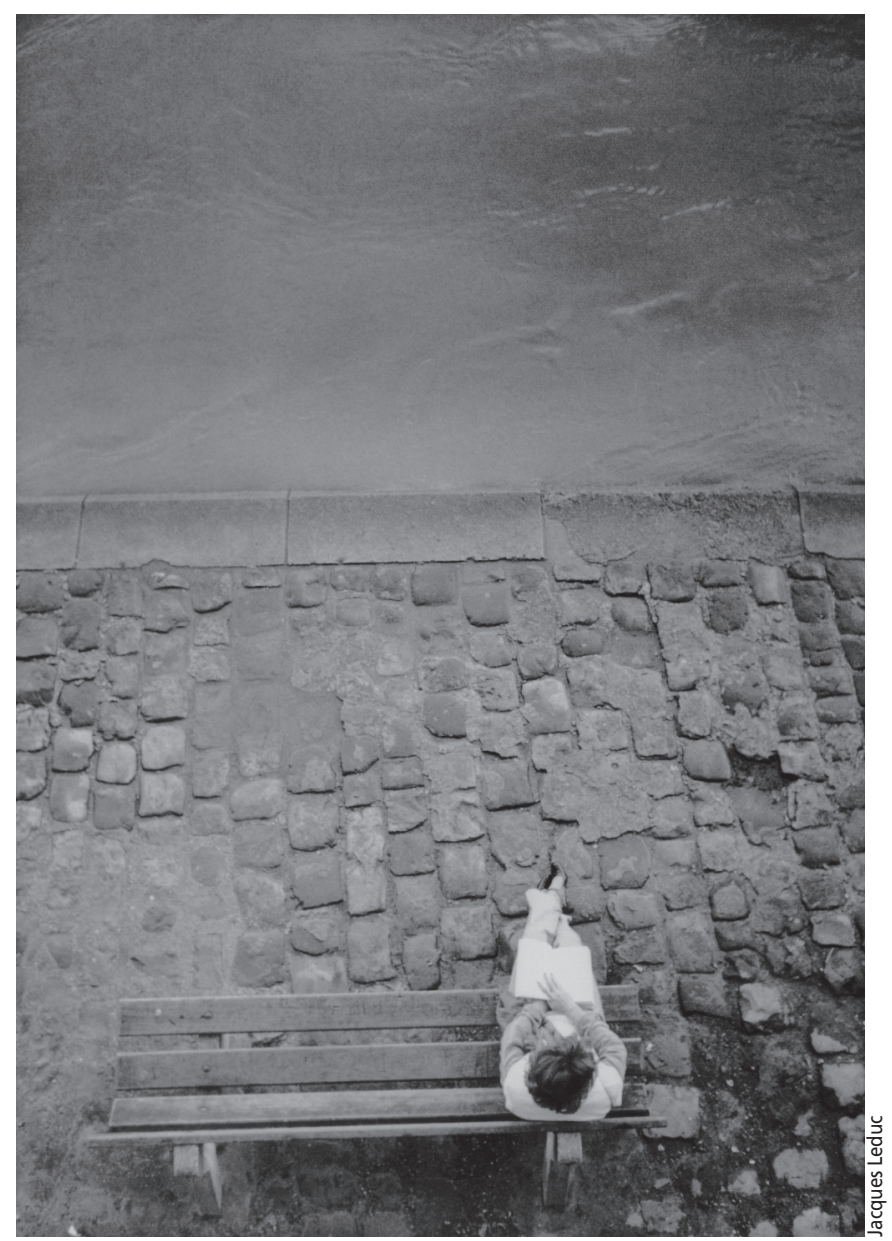


Très vite je gravite dans le monde interlope de l'argent. Un soir dans un bar, je fais une rencontre fortuite avec un gros dealer de coke et c'est parti! Je l'invite chez moi. À ce moment-là, j'habitais un penthouse en plein centre-ville, rien de moins. Lui, il y voit un bon stach pour ses kilos de coke et moi, je n'ai rien vu venir.

À 19 ans, je consomme avec classe, j'ai beaucoup de dope et d'ami(e)s. Je vends pour M.X. Je n'ai plus besoin de travailler, j'ai beaucoup d'argent et la vie est belle. Je n'ai pas connu de problèmes de dope à ce moment-là, car tant qu'il y en a, il n'y a pas de problème. C'est quand il n'y en a plus que les problèmes commencent. M. X et son stach sont disparus comme ils étaient venus. Je ne voulais pas que la fête prenne fin. J'étais dans mes débuts, là où tout est encore possible. J'avais gardé de mon travail quelques numéros de téléphone de clients qui m'avaient exprimé leurs fantasmes. C'est ainsi que j'ai commencé la prostitution, mais attention, de façon très professionnelle. Sauf que dans le monde illégal, tout bascule très, très vite. Ma consommation de free-base est devenue le pivot de mes désirs: j'oubliais de manger, de me laver et très vite j'ai perdu le penthouse et la voiture pour me retrouver dans un petit bordel à Ville SainteCatherine.
Je suis passée rapidement de la marge $I N$, celle qui est récréative, à la marge $O U T$, celle qui fait mal, celle qui détruit et isole.

Je n'ai pas besoin de vous dire que le monde merveilleux de la dope est violent, ingrat et malsain. Nous le savons tous, mais pourquoi en est-il ainsi? Je crois de plus en plus que c'est l'illégalité qui détruit, pas la dope en soi.

Pendant plus de quinze ans, j'ai eu ce goût amer de l'injection, mais surtout l'amertume que laissent la violence et l'injustice. Car que tu sois IN ou OUT, nous rêvons tous de réconfort et de sécurité. Les mille déceptions, les promesses des gens bienveillants, les abus de pouvoir des vendeurs de dope qui se croient des dieux. Toute cette violence que je me suis fait subir et pire, que j'ai fait subir aux autres. Ce trop plein de rage qui se retourne contre soimême. Je n'ai jamais été une personne violente, bien au contraire, j'avais un fond d'amour inculqué par ma famille, de bonnes valeurs.

Dans mes premières années de galère, j'ai adoré l'aventure que je vivais, la débrouillardise que suscitait mon besoin d'argent. Je ne manquais jamais de rien, j'étais jeune, belle et intelligente. Je regardais le reste de la société et me demandais comment ils faisaient pour aimer leur vie! Moi, ma vie était trépidante, grandiose. Je

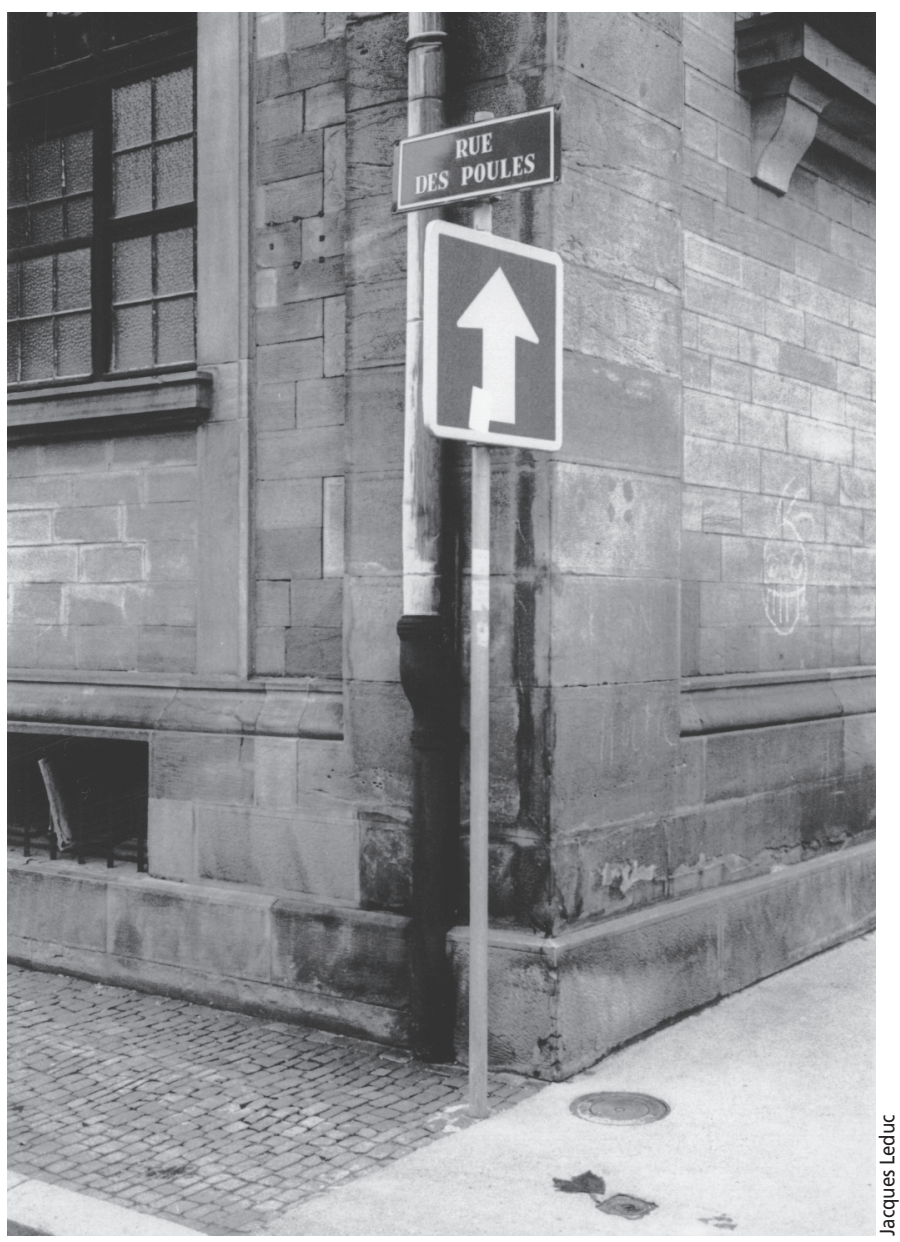

rencontrais des dizaines de personnes par jour et tous aimaient ma présence, mon corps et ma joie de vivre.

Plus le temps et les violences s'imprégnaient en moi, me tatouaient le corps, plus le désespoir prenait toute la place dans ma tête. J'agissais de plus en plus de façon irréfléchie, encaissais les coups, les déceptions et marchais la tête toujours plus basse. Je n'agissais plus, je réagissais. Je laissais tomber mes valeurs et ma fierté pour n'être qu'une junky, une pute. À mon tour, je suis devenue égoïste et avare. J'avais de foutues bonnes raisons. Un client venait de me battre, car je ne faisais pas telle ou telle pratique et on venait de me voler mon argent si durement sucé. Je n'avais plus de place sur mon corps meurtri pour me faire sentir ma propre déchéance. Quoi de mieux que de se tourner vers les innocents, à leur tour de les voir souffrir, ceux qui ne voulaient plus de moi, qui me méprisaient sans me connaître, ceux qui m'ont fermé la porte quand ils m'ont vue le visage plein de sang après m'être fait battre. Quelle conclusion pathétique, mais à ce moment-là c'était ma seule solution pour rester en vie. Seule je m'étais mise dans cette merde et j'en voulais au monde entier. Personne, pas même une droguée peut s'immuniser contre la souffrance du rejet. Moi qui étais fière d'être une junkie, me voilà dépérissant à vue d'œil. Chaque tentative de me sortir de cette merde ne faisait que me confirmer mon incapacité, mon mal de vivre, ma souffrance. Je ne m'en sortirais donc jamais...

C'est dans ces moments-là de désespoir extrême que je ne prêtais plus attention à rien. Je partageais mes seringues, à quoi bon, j'allais mourir dans cette galère anyway. Toujours la solitude gagnait $\mathrm{du}$ terrain, je ne pouvais plus supporter la paranoïa de mes congénères, toute cette méfiance que j'éprouvais en leur présence. Voici que mon merveilleux monde avait disparu pour ne faire place qu'à un grand vide, un trou noir. J'avais 31 ans et je m'injectais de la coke nuit et jour. Je n'avais plus de veine, ma chère héroïne, remplacée par l'hypocrite méthadone. Plus rien ne pouvait m'engourdir. J'étais verte, pesais 117 livres avec mes 5 pieds 7 pouces. Je faisais peur et pour couronner le tout, je n'avais plus une dent dans la gueule. J'étais morte.

La marginalité n'est pas une maladie incurable, pourtant nous la traitons ainsi. Pourquoi?

C'est mon anarchie et ma capacité d'émerveillement qui m'ont redonné l'espoir d'une vie meilleure. Je me suis rendue compte que j'étais devenue le résultat de la pensée des autres, que j'étais dans le carcan des idées préconçues de la société, celle qui me disait: "junkie un jour, junkie toujours...» 


\section{PARLONS UN PEU DE... LA PROSTITUTION}

«Mauvaises langues, écoutez: des gens crient! Dans les rues sombres, la nuit, se promènent une multitude de personnes différentes de celles que l'on croise tous les jours. Parmi ces gens, on retrouve évidemment les prostitués. [...] Il faut comprendre les raisons qui poussent ces personnes à agir de cette manière. Les prostitués ne font pas ce "métier" pour le plaisir ou parce qu'ils aiment ça! Ils sont prisonniers de l'enfer de la rue et nous, nous restons là, sans bouger. [...] Tous les égocentriques, tous les craintifs, arrêtez-vous une seconde. Regardons autour de nous: les travailleurs du sexe crient à l'aide. Allons vers eux. Dites-moi, préférezvous laisser des personnes dans la rue, comme des déchets?»

Louis Rocheleau-Lieutenant

«Il y a des préjugés concernant les jeunes prostitués. Est-ce vrai que les jeunes qui pratiquent la prostitution ne proviennent que de milieux défavorisés? Je ne crois pas. [...] On n'entre pas dans la prostitution du jour au lendemain: le processus est beaucoup plus long. L'influence que peuvent avoir des amis qui pratiquent déjà ce métier n'aide pas non plus. L'importance d'appartenir à un groupe d'amis peut souvent conduire un jeune à faire des choses qu'il ne ferait pas nécessairement en temps normal. [...] Bien sûr que les lieux propices à la prostitution sont les quartiers plus défavorisés, on ne peut pratiquer ce métier dans les grands quartiers riches. Mais ce n'est pas parce qu'ils travaillent dans un milieu défavorisé qu'ils proviennent nécessairement de ce milieu. [...] Il est trop facile de mettre en cause les parents qui éprouvent des problèmes financiers. Croyez-vous sincèrement que les problèmes de manque d'attention envers les enfants se retrouvent seulement dans les quartiers défavorisés? La prostitution ne dépend pas d'où l'on vit mais de ce que l'on vit!»

Catherine Germain tion des méfaits liés à l'usage des drogues. Je travaille aussi comme intervenante dans un site d'échange de seringues.

Plus le temps passe, plus je regagne une certaine confiance. Ce n'est pas toujours facile, je vis avec une multitude de souvenirs pas très roses. Aujourd'hui, c'est dans les petites choses que je vois les grands changements.

Jamais je ne pourrai vivre dans le contenu hermétique de la majorité, en me résignant au destin d'une société prévisible. Désormais, ma vie, mes expériences ont un sens.
Je n'étais pas que cela...

Un soir, je me suis retrouvée avec une arme qu'un client avait destinée à ma tête. Quand nous disons que les armes se retournent contre nous, c'est vrai. C'était le début de la fin. Mais je n'étais pas préparée à une telle transposition de mes souffrances. J'ai vomi violemment toute la violence subie, infligée. Je voulais mourir et j'ai failli tuer. J'ai continué la prostitution, mais je ne donnais plus aucun service, aucune douceur. J'étais un vrai cauchemar pour tous ceux qui se trouvaient sur mon chemin. J'ai reproduit les pires atrocités que l'on m'avait faites. J'ai encaissé pendant toutes ces années sans rien dire, en m'autodétruisant. Voici que plus rien ne pouvait retenir ma déchéance. Puis, vient l'inévitable. Je croyais avoir volé un client, mais c'était un vendeur. Personne ne peut nuire au monde merveilleux de la dope sans payer, même de sa vie. Voici qu'une fille se fait cribler de balles. Elle était jeune, belle et intelligente. J'avais la certitude que c'était une erreur, que c'était moi qu'il cherchait. C'est à ce moment-là que je m'en suis prise violemment à la bonne société, celle qui n'a rien à se reprocher, celle qui peut dénoncer. Je vous implore pourtant par mon geste de désespoir. Je vous fais cette violence avec une arme à feu. Mon aventure et mon incapacité de m'en sortir se sont retournées contre vous. J'étais anéantie. J'ai ressenti tout au long de mon long voyage vers la mort, les regards d'autrui se transformer au rythme de mes blessures. Plus j'avais mal, plus mon chemin devenait étroit. Mes choix disparaissaient avec mon image. Plus on avait peur de moi, plus je me rebellais et devenais méchante.

Enfermez-moi, je suis un danger en danger.

Le 19 mars 1999, je me fais arrêter pour huit vols à main armée et je reçois une sentence de quatre ans de pénitencier. Je ne peux vous décrire le sentiment de liberté que j'ai éprouvé à ce moment-là. Il y avait des années que je ne m'étais sentie aussi bien. Il m'a fallu la prison, sa solitude, ses structures de béton pour remettre de l'ordre dans ma vie de tumulte. L'écriture, le repos, deux longues années à prendre soin de moi. J'ai même profité de mon incarcération pour me défaire de mon dernier esclavage, la méthadone.

J'avais fait le tour du merveilleux monde des illusions. Ma marge était devenue toxique. Rien ne pouvait plus m'aider, ni l'amour des proches, car ça fait mal de toujours décevoir sans cesse. Ni mon propre amour, car il ne menait qu'à l'échec. Il était temps de me recréer une réalité. Une marge saine. Je suis remplie d'expériences et je veux construire. Car survivre à cette mort mérite de vivre...

Aujourd'hui je dis que j'ai un Ph.D. : private history of drugs. J'ai transposé ma déchéance en études et me suis mérité un diplôme bien gagné. J'évolue toujours dans le merveilleux monde de la marge, car pour moi c'est ici que la vie prend un sens. Aujourd'hui, j'essaie de construire. Je suis coordonnatrice d'un journal sur la réduc- 